PROCEEDINGS OF THE

AMERICAN MATHEMATICAL SOCIETY

Volume 138, Number 5, May 2010, Pages 1569-1574

S 0002-9939(10)10050-1

Article electronically published on January 8, 2010

\title{
REDUCTIONS OF IDEALS IN LOCAL RINGS WITH FINITE RESIDUE FIELDS
}

\author{
WILLIAM J. HEINZER, LOUIS J. RATLIFF, JR., AND DAVID E. RUSH
}

(Communicated by Bernd Ulrich)

\begin{abstract}
Let $I$ be a proper nonnilpotent ideal in a local (Noetherian) ring $(R, M)$ and let $J$ be a reduction of $I$; that is, $J \subseteq I$ and $J I^{n}=I^{n+1}$ for some nonnegative integer $n$. We prove that there exists a finite free local unramified extension ring $S$ of $R$ such that the ideal $I S$ has a minimal reduction $K \subseteq$ $J S$ with the property that the number of elements in a minimal generating set of $K$ is equal to the analytic spread of $K$ and thus also equal to the analytic spread of $I$.
\end{abstract}

\section{INTRODUCTION}

Let $I$ be an ideal in a Noetherian ring $R$. Northcott and Rees in [6] introduced the concept of a reduction of $I$, where an ideal $J$ of $R$ is a reduction of $I$ if $J \subseteq I$ and $J I^{n}=I^{n+1}$ for all large integers $n$. If $J$ is a reduction of $I$, then $I$ is integral over $J$. As remarked by Swanson and Huneke in [9, page 5], reductions are an extremely useful tool for integral closure.

Let $I$ be an ideal in a local ring $(R, M)$ and let $t$ be an indeterminate. The analytic spread $\ell(I)$ of the ideal $I$ is the dimension of the graded ring

$$
R[I t] / M R[I t]=R / M \oplus I / M I \oplus I^{2} / M I^{2} \oplus \cdots .
$$

Northcott and Rees prove in [6] that every reduction of $I$ requires at least $\ell(I)$ generators; moreover, if $R / M$ is infinite, then there exist reductions of $I$ generated by $\ell(I)$ elements.

In the case where $(R, M)$ is a local ring and $R / M$ is finite, Northcott and Rees prove in [7] that if $\operatorname{dim} R=d>0$, if $\operatorname{char}(R)=\operatorname{char}(R / M)$, and if $I$ is $M$-primary, then there always exist positive integers $r_{i}, i=1, \ldots, d$, and elements $b_{i} \in I^{r_{i}}$ such that $I^{m}=b_{1} I^{m-r_{1}}+\cdots+b_{d} I^{m-r_{d}}$ for at least one positive integer $m \geq$ $\max \left\{r_{i} \mid i=1, \ldots, d\right\}$. The elements $b_{1}, \ldots, b_{d}$ are called a reduction of $I$ of type $\left[r_{1}, \ldots, r_{d}\right]$. Northcott and Rees prove in [7, Theorem 4] that there exists a positive integer $n$, depending on $I$, such that $I^{n}$ has a reduction $c_{1}, \ldots, c_{d}$ of type $[1, \ldots, 1]$, so $\left(c_{1}, \ldots, c_{d}\right) R$ is a reduction of $I^{n}$.

Another appoach often used for dealing with reductions of an ideal $I$ in a local ring $(R, M)$ in the case where $R / M$ is finite is to replace $(R, M)$ and $I$

Received by the editors February 13, 2009, and, in revised form, May 26, 2009.

2000 Mathematics Subject Classification. Primary 13A15, 13E05, 13 H10.

Key words and phrases. Minimal reduction, analytic spread, finite free local unramified extension, Rees valuation rings, projective equivalence of ideals. 
with $(R(X), M R(X))$ and $I R(X)$, where $X$ is an indeterminate and $R(X)=$ $R[X]_{M R[X]}$; cf. [9, Section 8.4]. Then $R(X) / M R(X)$ is isomorphic to the infinite field $(R / M)(X)$, so $I R(X)$ has a minimal reduction that is generated by $\ell(I R(X))$ elements. Since $\ell(I R(X))=\ell(I)$, this is often helpful for deducing certain things about $I$ and $R$.

In the present paper, we consider a different approach to reductions in the case where $R / M$ is finite. We use the classical concept of unramified extensions as in the following definition.

Definition 1.1. A quasi-local ring $(S, N)$ is unramified over a quasi-local ring $(R, M)$ in the case where $R$ is a subring of $S, N=M S$, and $S / N$ is separable over $R / M$. If $S$ is an extension ring of a ring $R$ and $q$ is a prime ideal of $S$, then $q$ is unramified over $q \cap R$ in the case where $S_{q}$ is unramified over $R_{q \cap R}$.

Let $I$ be a nonnilpotent ideal in a local ring $(R, M)$. We show in Theorem 3.1 that there exists a finite free local extension ring $(S, N)$ of $R$ such that $S$ is unramified over $R$ and such that $I S$ has a minimal reduction that is generated by $\ell(I S)=\ell(I)$ elements. In Example 2.3 we exhibit, for each finite field $F$, a Cohen-Macaulay local ring $(R, M)$ of dimension two with $R / M=F$ such that the associated graded ring $G(M)$ is Cohen-Macaulay and such that $M$ has no reductions generated by analytically independent elements.

We were motivated to consider reductions of ideals in local rings with finite residue fields by our work in [4] on generating sets of ideals and Rees valuation rings. By considering unramified finite integral extensions we have been able to extend results obtained in the papers $[2]$ and $[3$ in the case of infinite residue fields to the case of finite residue fields. The main result here, Theorem 3.1, gives another instance where some desirable behavior in a local ring $(R, M)$ which usually depends on $R / M$ being infinite can be recovered in the finite residue field case by passing to a finite free local unramified extension ring $S$ of $R$. We present in Theorem 4.1 an application of Theorem 3.1

Our notation is mainly as in [5] and [10].

\section{Analytic indePendence And a motivating eXample}

We recall several definitions from [6].

Definition 2.1. Let $I$ be an ideal of a local ring $(R, M)$. Then:

(2.1.1) Elements $b_{1}, \ldots, b_{g}$ in $I$ are analytically independent in $I$ in the case when the following holds: if $f\left(X_{1}, \ldots, X_{g}\right) \in R\left[X_{1}, \ldots, X_{g}\right]$ is a form of arbitrary positive degree $n$ such that $f\left(b_{1}, \ldots, b_{g}\right) \in M I^{n}$, then $f\left(X_{1}, \ldots, X_{g}\right) \in$ $M\left[X_{1}, \ldots, X_{g}\right]$; that is, all the coefficients of $f\left(X_{1}, \ldots, X_{g}\right)$ are in $M$.

(2.1,2) Elements $b_{1}, \ldots, b_{g}$ in $R$ are analytically independent in the case when the following holds: if $f\left(X_{1}, \ldots, X_{g}\right) \in R\left[X_{1}, \ldots, X_{g}\right]$ is a form of arbitrary positive degree $n$ such that $f\left(b_{1}, \ldots, b_{g}\right)=0$, then $f\left(X_{1}, \ldots, X_{g}\right) \in M\left[X_{1}, \ldots, X_{g}\right]$; that is, all the coefficients of $f\left(X_{1}, \ldots, X_{g}\right)$ are in $M$.

(2.1,3) An ideal $J \subseteq I$ is said to be a minimal reduction of $I$ if $J$ is a reduction of $I$ and no ideal strictly contained in $J$ is a reduction of $I$.

(2.1,4) Let $\mu(I)$ denote the number of elements in a minimal generating set of the ideal $I$. 
We collect in Remark 2.2 several facts about analytic independence and reductions.

Remark 2.2. Let $(R, M)$ be a local ring and let $I$ be an ideal of $R$.

(2.2.1) Elements $b_{1}, \ldots, b_{g}$ in $R$ are analytically independent if and only if they are analytically independent in the ideal $\left(b_{1}, \ldots, b_{g}\right) R$ that they generate.

(2.2.2) If $b_{1}, \ldots, b_{g}$ are analytically independent in $I$, then $\left\{b_{i}, \ldots, b_{g}\right\}$ is a minimal generating set of the ideal $\left(b_{1}, \ldots, b_{g}\right) R$.

(2.2.3) In general there is no unique minimal reduction of the ideal $I$, but if $J$ is a reduction of $I$, then there exists at least one ideal $K \subseteq J$ such that $K$ is a minimal reduction of $I$ [9, Theorem 8.3.6].

$(2.2,4)$ If $J$ is a minimal reduction of $I$, then elements in a minimal generating set for $J$ are part of a minimal generating set for $I$ [9, Proposition 8.3.3].

(2.2.5) If $J$ is a reduction of $I$ and $\mu(J)=\ell(I)$, then $J$ is a minimal reduction of $I$ and every minimal generating set of $J$ consists of analytically independent elements [9. Corollary 8.3.5].

$\left(2.2\right.$.6) There exists a positive integer $n$ such that $I^{n}$ has a reduction generated by $\ell(I)$ elements [9, Proposition 8.3.8].

2.2.7) If $(R, M)$ has an infinite residue field, then every minimal reduction $J$ of $I$ is generated by $\ell(I)=\ell(J)$ elements and these elements are analytically independent [6. Lemma 2, p. 149].

With Remark 2.2.7 in mind, in Example 2.3 we exhibit a Cohen-Macaulay local ring $(R, M)$ of dimension two, so $\ell(M)=2$, such that $M$ has no (minimal) reduction generated by two elements.

Example 2.3. Let $F$ be an arbitrary finite field. There exists a Cohen-Macaulay local ring $(R, M)$ of dimension two with $R / M=F$ such that the associated graded ring

$$
G(M)=R / M \oplus M / M^{2} \oplus M^{2} / M^{3} \oplus \cdots
$$

is Cohen-Macaulay and such that $M$ has no reductions generated by analytically independent elements.

Proof. Let $n$ denote the number of nonzero elements in $F$, let $\left\{X_{i}\right\}_{i=1}^{n+3}$ be indeterminates and let $S$ denote the polynomial ring $F\left[\left\{X_{i}\right\}_{i=1}^{n+3}\right]$. For each pair of integers $j$ and $k$ with $1 \leq j<k \leq n+3$, let $P_{j k}$ denote the prime ideal of $S$ generated by the $n+1$ variables $X_{i} \in\left\{X_{i}\right\}_{i=1}^{n+3}$ with $i \notin\{j, k\}$. Let $I$ denote the intersection of the $\left(\begin{array}{c}n+3 \\ 2\end{array}\right)$ prime ideals $P_{j k}$ and let $T=S / I$. Regarding the polynomial ring $S$ as a graded ring with each $X_{i}$ of degree one, it is clear that

$$
T=\bigoplus_{i \geq 0} T_{i}=F\left[T_{1}\right]
$$

is a homogeneous graded ring of dimension two. Let $N=\bigoplus_{i>1} T_{i}$ denote the graded maximal ideal of $T$ and let $R=T_{N}$ and $M=N T_{N}$. Then $(R, M)$ is a local ring of dimension two and the associated graded ring $G(M) \cong T$. To prove that $R$ is Cohen-Macaulay, it suffices to show that $T$ is Cohen-Macaulay, and to prove that $M$ has no reductions generated by analytically independent elements, it suffices to prove that $T$ has no homogeneous system of parameters consisting of linear forms. 
Notice that the minimal prime ideals of $T$ are precisely the prime ideals $P_{j k} T$ and that $T / P_{j k} T=S / P_{j k} \cong F\left[X_{j}, X_{k}\right]$ and $M / P_{j k} R$ is the maximal ideal of the localized polynomial ring $F\left[X_{j}, X_{k}\right]_{\left(X_{j}, X_{k}\right)}$. Therefore $M$ has $\left(\begin{array}{c}n+3 \\ 2\end{array}\right)$ Rees valuation rings with exactly one Rees valuation ring corresponding to each minimal prime of $R$ or, equivalently, of $T$. The Rees valuation ring of $M$ corresponding to $P_{j k}$ is described by the order valuation on the polynomial ring $F\left[X_{j}, X_{k}\right]$. The $\operatorname{ring} T$ is the Stanley-Reisner ring associated to the simplicial complex $\Delta$ on a vertex set $V=\left\{v_{1}, \ldots, v_{n+3}\right\}$, where $\operatorname{dim} \Delta=1$ and where each edge $\left\{v_{j}, v_{k}\right\}$ with $1 \leq j<$ $k \leq n+3$ is in $\Delta$. It is clear that $\Delta$ is connected. Since $\operatorname{dim} \Delta=1$, it follows that $\Delta$ is shellable and $T=F[\Delta]$ is a Cohen-Macaulay ring; cf. [1, Exercise 5.1.26, page 222]. Let $x_{i}$ denote the image of $X_{i}$ in $T_{1}$. Notice that $T_{1}$ is a vector space over $F$ of dimension $n+3$ and the $x_{i}$ form an $F$-basis for $T_{1}$. Suppose there exist $f, g \in T_{1}$ that form a system of parameters for $T$. Since $T$ is Cohen-Macaulay, $f, g$ is a regular sequence on $T$, and for each nonzero $a \in F$, the element $f-a g$ is regular on $T$. Therefore $f-a g$ is not in any minimal prime of $T$. This means that

$$
f-a g=b_{1} x_{1}+\cdots+b_{n+3} x_{n+3},
$$

where the $b_{i} \in F$ and at most one of the $b_{i}=0$. Both $f$ and $g$ also have this property. Therefore $f$ and $g$ both have a nonzero coefficient of $x_{i}$ for at least $n+1$ of the $x_{i}$, and we may assume this is true for $x_{1}, \ldots, x_{n+1}$. Then for each $i \in\{1, \ldots, n+1\}$ there exists a nonzero $a_{i} \in F$ such that $f-a_{i} g$ has zero as the coefficient of $x_{i}$. If $c_{i}$ is the coefficient of $x_{i}$ for $f$ and $d_{i}$ is the coefficient of $x_{i}$ for $g$, then $f-\frac{c_{i}}{d_{i}} g$ has zero as the coefficient of $x_{i}$. Since $F$ has only $n$ nonzero elements, there exists a nonzero element $a \in F$ such that $f-a g$ has zero as the coefficient of $x_{i}$ for at least two $i$ with $1 \leq i \leq n+1$. This implies that $f-a g$ is in some minimal prime of $T$, which contradicts the fact that $f-a g$ is regular on $T$.

\section{REDUCTIONS IN LOCAL RINGS With FINITE RESIDUE FIELDS}

Examples such as Example 2.3 motivate our consideration in Theorem 3.1 of reductions of ideals in a local ring $(R, M)$ that have a finite residue field.

Theorem 3.1. Let $I$ be a nonnilpotent ideal of a local (Noetherian) ring $(R, M)$. Let $X$ be an indeterminate, and assume that $\ell\left(\operatorname{IR}[X]_{M R[X]}\right)=g$. Let $J$ be a reduction of $I$ and let $\mu(J)=h$. Then $h \geq g$, and there exists a simple finite free local unramified extension ring $(S, N)$ of $R$ such that $I S$ has a minimal reduction $K \subseteq$ $J S$ with $\mu(K)=\ell(K)=g=\ell(K S(X))$.

Proof.1 If $R / M$ is infinite, then Remark 2.2.7 implies the conclusion holds with $S=R$. Also, if $h=g$, then Remark 2.2.5 implies the conclusion holds with $S=R$. Thus it may be assumed that the field $R / M=F$ is finite and that $h>g$.

Let $R^{*}=R[X]_{M R[X]}$. Then $R^{*} /\left(M R^{*}\right) \cong F(X)$ is an infinite field, so there exist elements $c_{1}(X), \ldots, c_{g}(X)$ in $J R^{*}$ such that $L=\left(c_{1}(X), \ldots, c_{g}(X)\right) R(X)$ is a minimal reduction of $I R(X)$, say $I^{n+1} R^{*}=L I^{n} R^{*}$. It may be assumed that $c_{1}(X), \ldots, c_{g}(X) \in R[X]$. There exists a polynomial $r(X) \in R[X] \backslash M R[X]$ such that

$$
r(X) I^{n+1} R[X] \subseteq L I^{n} R[X] \subseteq I^{n+1} R[X] .
$$

\footnotetext{
${ }^{1}$ We thank the referee for suggesting the proof given here. It is shorter and more direct than our original proof.
} 
Let an overbar denote residue classes modulo $M$. Then $\bar{r}(X)$ is a nonzero polynomial in $F[X]$. Let $x$ be an element in the algebraic closure of the finite field $F$ with the property that the degree of the field extension $F(x) / F$ is greater than the degree of the polynomial $\bar{r}(X)$. Thus $\bar{r}(x) \neq 0$. Let $G=F[x]=F[X] /(\bar{f}(X) R[X])$, where $\bar{f}(X) \in F[X]$ is the minimal monic polynomial of $x$ over $F$.

Let $f(X)$ be a monic pre-image of $\bar{f}(X)$ in $R[X]$ and let $S=R[X] /(f(X) R[X])$, so $S=R[y]$ with $y=X+(f(X)) R[X]$. Then $S /(M S) \cong F[x]=G$. It follows that $S$ is a simple finite free extension ring of $R$ with $M S$ its unique maximal ideal and $S$ is unramified over $R$ as in Definition 1.1.

Let $K=\left(c_{1}(y), \ldots, c_{g}(y)\right) S$, where the $c_{i}(X)$ are as in the second paragraph of this proof, so $K=L /(f(X) R[X])$. It follows from $\left(^{*}\right)$ that $K$ is a minimal reduction of $I S$ contained in $J S$ and that $\mu(K)=\ell(K)=g=\ell(K S(X))$.

\section{An APPLICATION CONCERNING PROJECTIVE EQUIVALENCE}

Let $I$ be a regular proper ideal of the Noetherian ring $R$; that is, $I$ contains a regular element of $R$ and $I \neq R$. An ideal $J$ of $R$ is projectively equivalent to $I$ if there exist positive integers $m$ and $n$ such that $\left(I^{m}\right)_{a}=\left(J^{n}\right)_{a}$, where $K_{a}$ is the integral closure in $R$ of an ideal $K$ of $R$. Let $\mathbf{P}(I)$ denote the set of integrally closed ideals that are projectively equivalent to $I$.

The set Rees $I$ of Rees valuation rings of $I$ is a finite set of rank one discrete valuation rings (DVRs) that determine the integral closure $\left(I^{n}\right)_{a}$ of $I^{n}$ for every positive integer $n$ and is the unique minimal set of DVRs having this property; cf. [9. Section 10.1]. If $\left(V_{1}, N_{1}\right), \ldots,\left(V_{n}, N_{n}\right)$ are the Rees valuation rings of $I$, then the integers $\left(e_{1}, \ldots, e_{n}\right)$, where $I V_{i}=N_{i}^{e_{i}}$, are the Rees integers of $I$.

Theorem 4.1 illustrates how Theorem 3.1 may be applied. The issues considered in Theorem 4.1] are developed further in [2, 3] and [4.

Theorem 4.1. Let I be an $M$-primary regular ideal of a quasi-unmixed local ring $(R, M)$ of dimension $d$.

(4.1,1) If the greatest common divisor of the Rees integers of $I$ is a unit in $R$, then there exists a finite free extension ring $A$ of $R$ and an ideal $J$ of $A$ such that $\mathbf{P}(I A)=\mathbf{P}(J)=\left\{\left(J^{n}\right)_{a} \mid n \geq 1\right\}$. Further, if $R$ is an integral domain and $z$ is a minimal prime ideal of $A$, then the ideal $J_{1}=(J+z) / z$ in the integral domain $A_{1}=A / z$ is such that $\mathbf{P}\left(I A_{1}\right)=\mathbf{P}\left(J_{1}\right)=\left\{\left(J_{1}{ }^{n}\right)_{a} \mid n \geq 1\right\}$.

(4.1,2) If the least common multiple of the Rees integers of $I$ is a unit in $R$, then there exists a finite free extension ring $A$ of $R$ and a radical ideal $J$ of $A$ such that $\mathbf{P}(I A)=\mathbf{P}(J)=\left\{\left(J^{n}\right)_{a} \mid n \geq 1\right\}$ and $J V=N$ for all Rees valuation rings $(V, N)$ of $J$. Further, if $R$ is an integral domain and $z$ is a minimal prime ideal of $A$, then $J_{1}=(J+z) / z$ is a radical ideal in the integral domain $A_{1}=A / z$ and $\mathbf{P}\left(I A_{1}\right)=\mathbf{P}\left(J_{1}\right)=\left\{\left(J_{1}{ }^{n}\right)_{a} \mid n \geq 1\right\}$ and $J_{1} V_{1}=N_{1}$ for all Rees valuation rings $\left(V_{1}, N_{1}\right)$ of $J_{1}$.

Proof. For 4.1,1), by Theorem 3.1 there exists a finite free local unramified extension ring $(S, N)$ of $R$ such that $I S$ has a minimal reduction $K \subseteq I S$ with $\mu(K)$ $=\ell(K)=d=\ell(K S(X))$. Then $\mathbf{P}(I S)=\mathbf{P}(K)$ and $K=\left(b_{1}, \cdots, b_{d}\right) S$, where the $b_{i}$ are analytically independent. Since $S$ is unramified over $R$, it follows that $I S$ has the same Rees integers as $I$, with possibly some of the Rees integers of $I S$ occurring more times. Also, $I S$ and $K$ have the same Rees valuation rings and 
Rees integers, since $K$ is a reduction of $I S$. Further, $S$ is quasi-unmixed, by 8 Corollary 2.14 and Theorem 3.1], since $R$ is quasi-unmixed and $S$ is a finite free extension ring of $R$. Thus we may replace $(R, M)$ by $(S, N)$ and $I$ by $K$. Since $(S, N)$ is quasi-unmixed, $b_{i} V=I V$ for each $V \in \operatorname{Rees} K=\operatorname{Rees} I S$ and each $i=$ $1, \ldots, d$ by [9, Theorem 10.26]. Therefore (4.11) follows from [2, Theorem 2.5].

The proof of (4.1.2) is similar, but use [3, Theorem 3.7] in place of [2, Theorem 2.5].

\section{REFERENCES}

1. W. Bruns and J. Herzog, Cohen-Macaulay Rings, revised edition, Cambridge Univ. Press, Cambridge 1998. MR1251956 (95h:13020)

2. C. Ciupercă, W. J. Heinzer, L. J. Ratliff, Jr., and D. E. Rush, Projectively full ideals in Noetherian rings (II), J. Algebra 305 (2006), 974-992. MR2266864 (2007m:13010)

3. W. J. Heinzer, L. J. Ratliff, Jr., and D. E. Rush, Projectively full radical ideals in integral extension rings, J. Algebra 317 (2007), 833-850. MR2362944 (2009b:13012)

4. W. J. Heinzer, L. J. Ratliff, Jr., and D. E. Rush, Bases of ideals and Rees valuation rings, J. Algebra 323 (2010), 839-853.

5. M. Nagata, Local Rings, Interscience, John Wiley, New York, 1962. MR0155856 (27:5790)

6. D. G. Northcott and D. Rees, Reductions of ideals in local rings, Proc. Cambridge Philos. Soc. 50 (1954), 145-158. MR0059889 (15:596a)

7. D. G. Northcott and D. Rees, A note on reductions of ideals with an application to the generalized Hilbert function, Proc. Cambridge Philos. Soc. 50 (1954), 353-359. MR0062115 $(15: 929 \mathrm{e})$

8. L. J. Ratliff, Jr., On quasi-unmixed local domains, the altitude formula, and the chain condition for prime ideals (I), Amer. J. Math. 91 (1969), 508-528. MR0246867 (40:136)

9. I. Swanson and C. Huneke, Integral Closure of Ideals, Rings and Modules, Cambridge Univ. Press, Cambridge, 2006. MR.2266432 (2008m:13013)

10. O. Zariski and P. Samuel, Commutative Algebra, Vol. 1, D. Van Nostrand, Princeton, NJ, 1958. MR0090581(19:833e)

Department of Mathematics, Purdue University, West Lafayette, Indiana 47907-1395

E-mail address: heinzer@math.purdue.edu

Department of Mathematics, University of California, Riverside, California 925210135

E-mail address: ratliff@math.ucr.edu

Department of Mathematics, University of California, Riverside, California 925210135

E-mail address: rush@math.ucr.edu 